\title{
NOTAS VARIAS SOBRE HELICONIA RODRIGUEZII (HELICONIACEAE) DE COSTA RICA
}

\author{
CARlos O. Morales \\ Escuela de Biología, Universidad de Costa Rica. 2060 San José, Costa Rica, \\ y Jardín Botánico Lankester. Apdo. 1031-7050 Cartago, Costa Rica
}

\begin{abstract}
Heliconia rodriguezii was described in 1982, but all the Costa Rican specimens were at the USJ Herbarium. Isotypes were distributed to other herbaria lately in May and June 2002. Moreover the herbarium acronyms for the isotypes are erroneously cited in the original publication. Notes on distribution, ecology, and morphology of this rare, endemic, and poorly known species are given.

RESUMEN. Heliconia rodriguezii fue descrita en 1982, pero todos los especímenes de Costa Rica se hallaban en el Herbario USJ. Los isotipos se enviaron a otros herbarios hasta mayo y junio de 2002. Además, los acrónimos de herbarios para los isotipos se citan mal en la publicación original. Se anotan datos sobre distribución, ecología y morfología de esta especie endémica, escasa y poco conocida.
\end{abstract}

Palabras clave / Key words: Heliconiaceae, Heliconia rodriguezii, especímenes tipo, Costa Rica

En Costa Rica se conocen unas 42 especies nativas de Heliconia. De éstas, unas once especies (ca. $26 \%$ ) son endémicas de este país y es probable que aún se describan algunas especies nuevas. Aunque en la literatura aparecen unos 450 nombres de especies, variedades e híbridos, Berry \& Kress (1991) sitúan entre 200 y 250 el número total de especies de Heliconia. Así, la diversidad registrada en Costa Rica representa entre 15 y $20 \%$ de las especies del género. Stiles (1985) señala que el centro de diversificación se halla en la Sudamérica tropical. En Costa Rica, según las observaciones del mismo autor, la mayor diversidad de Heliconia se concentra en la vertiente atlántica al pie de las cordilleras Central y de Talamanca, entre 100 y $700 \mathrm{~m}$ de altitud, y en la Península de Osa y áreas vecinas.

En lugar de estudiar material de herbario, Daniels \& Stiles (1979) y Stiles (1975, 1979, 1980, 1982, 1985) observaron y describieron las heliconias in vivo. De este modo, su trabajo ha sido crítico para entender tanto la taxonomía heliconiácea costarricense como la estrecha relación ecológica y evolutiva existente entre estas plantas y dos grupos de aves: colibríes, que polinizan todas las especies de Heliconia de Costa Rica, y aves frugívoras, que dispersan frutos y semillas.

Una de las especies menos conocidas, Heliconia rodriguezii F. G. Stiles (1982: 222), dedicada al insigne botánico, artista y humanista costarricense Rafael Lucas Rodríguez, fue descrita de La Montura, en el Parque Nacional Braulio Carrillo, donde, según el autor, la especie es común en laderas y barrancas boscosas y se halla a menudo en claros ocasionados por caída de árboles. Los datos del tipo indican lo siguiente: "Stiles 80-27, forest understory at La Montura, el. 1050 m, Parque Nacional Braulio Carrillo, Provincia San José, Costa Rica, 27 July 1980; UCR (holotype), MNCR, FMNH, USNM."

Tenemos dos problemas con estos datos:

1) Los acrónimos de los herbarios citados son incorrectos. Sin duda, "UCR" se refiere al Herbario de la Universidad de Costa Rica (USJ), "MNCR" alude al Herbario Nacional (CR), en el Museo Nacional de Costa Rica, "FMNH" es el Herbario del Field Museum of Natural History (F) en Chicago, EE.UU., y "USNM" tiene que ser el United States National Herbarium (US), en Washington, EE.UU.

2) En USJ observé siete ejemplares completos del tipo de Heliconia rodriguezii, lo que claramente revela que los isotipos citados por Stiles nunca fueron enviados a los respectivos herbarios. Esto ya fue confirmado por curadores de los herbarios CR, F y US.

En mayo y junio de 2002 han sido enviados ejemplares isotipos a los herbarios CR, F, M, ULM y US, 


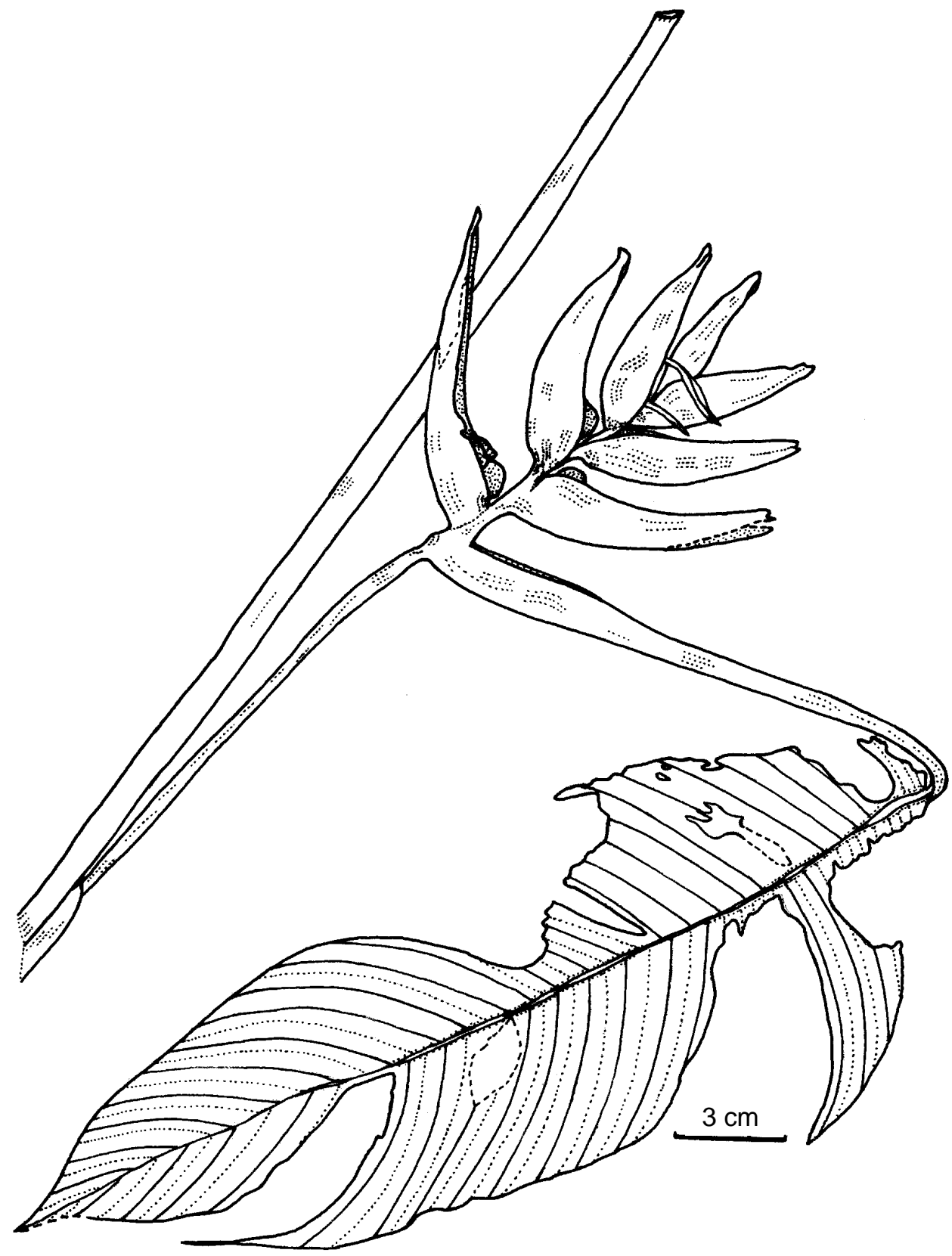

Figura. 1. Heliconia rodriguezii F.G. Stiles. Isotipo (USJ). Dibujo del autor.

mientras que el holotipo (así anotado por Stiles) y un isotipo permanecen en USJ.

Según Stiles, las especies más emparentadas con H. rodriguezii (Fig. 1) son H. gracilis G.S. Daniels \& F.G. Stiles y H. ignescens G.S. Daniels \& F.G. Stiles.
La primera se diferencia de éstas dos por el hábito más musoide, vástago (pseudotallo) más grande y más robusto, hojas más grandes e inflorescencias más anchas y voluminosas. Stiles considera que, morfológicamente, $H$. rodriguezii es un eslabón entre las dos especies mencionadas. Uno de los isotipos (F) 
indica: "Planta de $1.5 \mathrm{~m}$ de altura. Inflorescencia roja, flor amarillenta." Kress (in Hammel et al., inéd.) señala que las brácteas medias poseen una superficie externa glabra, roja a rojo-anaranjada. Además, indica en su clave de las heliconias de Costa Rica que $H$. ignescens tiene láminas foliares menores que $50 \mathrm{~cm}$ de largo y brácteas cincinales anaranjadas, mientras que $H$. rodriguezii muestra láminas foliares mayores que $50 \mathrm{~cm}$ de largo y brácteas cincinales rojas. Esta especie, que aparentemente florece la mayor parte del año, se considera rara y es endémica en el Parque Nacional Braulio Carrillo, en las laderas atlánticas de la Cordillera Central, entre 800 y $1200 \mathrm{~m}$ de elevación, justamente en el área de mayor diversidad del género Heliconia en Costa Rica. Stiles (1982) incluye una fotografía de la inflorescencia de $H$. rodriguezii en blanco y negro. En Berry \& Kress (1991: 167) aparece una fotografía en colores. En esta última obra, así como en Daniels \& Stiles (1979), aparecen también fotografías en colores de las dos especies emparentadas, mencionadas anteriormente.

\section{OTROSESPECÍMENESVISTOS:}

F.G. Stiles 80-32 (USJ). Localidad típica. 27 de julio de 1980. "... flor amarilla."

F.G. Stiles 83-68 (USJ). "Braulio Carrillo". 8 de abril de 1983.

F.G. Stiles s.n. (USJ-30864). Localidad típica, $1020 \mathrm{~m}$ de altitud. 23 de abril de 1989. "Brácteas rojas; flores amarillas."

Es indudable que $H$. rodriguezii ha permanecido desconocida para la mayoría de botánicos que trabajan en Costa Rica, en parte debido a que los isotipos no se distribuyeron. Además, muy pocos botánicos han recolectado esta especie después de Stiles. Así, por ejemplo, antes de mayo de 2002 no había ningún ejemplar en CR. En la base de datos TRÓPICOS, del Jardín Botánico de Missouri, se cita un solo ejemplar: B.A. Loiselle 224 (MO) [Heredia, $1530 \mathrm{~m}, 10^{\circ} 14^{\prime} \mathrm{N}$, $84^{\circ} 05^{\prime} \mathrm{W}$, January $1987,1-1.5 \mathrm{~m}$; red upright bracts; inflorescence and unripe fruit in January]. La base de datos ATTA, del Instituto Nacional de Biodiversidad, incluye asimismo un solo ejemplar: P.J.M. Maas 7809 (INB) [área del Río Sucio, Parque Nacional Braulio Carrillo]. Durante muchos años esta especie ni siquiera apareció en algunas listas de plantas de Costa Rica.

\section{LITERATURA CITADA}

Berry, F. \& Kress, W.J. 1991. Heliconia, an identification guide. Washington/London. Smithsonian Institution Press. 334 p.

Daniels, G.S. \& Stiles, F.G. 1979. The Heliconia taxa of Costa Rica. Keys and descriptions. Brenesia 15 (Supl.): 1-150.

Instituto Nacional de Biodiversidad (INBio). 2002. Sistema ATTA. http://atta.inbio.ac.cr/

Kress, W.J. Heliconiaceae. In: Hammel, B.E., Zamora, N. \& Grayum, M.H. (eds.). Manual de Plantas de Costa Rica. Vol. 1: Monocotiledóneas. Heredia, INBio, y St. Louis, Missouri Bot. Gard. (manuscrito).

Missouri Botanical Garden. 2002. TROPICOS. http://mobot.mobot.org/W3T/Search/vast.html

Stiles, F.G. 1975. Ecology, flowering phenology, and hummingbird pollination of some Costa Rican Heliconia species. Ecology 52: 285-301.

Stiles, F.G. 1979. Notes on the natural history of Heliconia (Musaceae) in Costa Rica. Brenesia 15 (Supl.): 151180.

Stiles, F.G. 1980. Further data on the genus Heliconia (Musaceae) in northern Costa Rica. Brenesia 18: 147154.

Stiles, F.G. 1982. Taxonomic and distributional notes on Costa Rican Heliconia (Musaceae), II: Parque Nacional Braulio Carrillo, Cordillera Central. Brenesia 19/20: 221-230.

Stiles, F.G. 1985. Aspectos de la ecología, distribución y evolución del género Heliconia en Costa Rica. In: D'Arcy, W.G. \& Correa, Mireya D. (eds.). The botany and natural history of Panama / La botánica e historia natural de Panamá. p. 273-285. Missouri Botanical Garden. 